\title{
Some properties of a kind of generalized Teodorescu operator in Clifford analysis
}

\section{Liping Wang*}

"Correspondence: wlpxjj@163.com
College of Mathematics and
Information Science, Hebei Normal
University, Shijiazhuang, Hebei
province 050024, P.R. China

\begin{abstract}
First, a kind of generalized Teodorescu operator which is related to the k-regular functions are defined. Then the boundedness and the Hölder continuity of the generalized Teodorescu operator are discussed. Finally, the stability and error estimate of the generalized Teodorescu operator are given when the boundary surface of the integral domain $\Omega$ is perturbed.
\end{abstract}

Keywords: Clifford analysis; $k$-regular functions; generalized Teodorescu operator; Hölder continuity; stability

\section{Introduction}

The Clifford algebra $\mathcal{A}_{n}(R)$ was established by Clifford [1] in 1878, which is the extension of complex numbers, quaternions, and exterior algebras. It is an associative and incommutable algebra structure. And it possesses both theoretical and applicable value to many fields, such as quantum mechanics, quantum field theory [2], projective geometry, computer graphics [3-5], neural network theory [6,7], and so on. Clifford analysis is an important branch of modern analysis, which studies functions defined on $R^{n}$ with their values in Clifford algebra space $\mathcal{A}_{n}(R)$. Clifford analysis is an important tool of modern mathematics and physics. In addition, Clifford analysis is now a widely studied field [8]: function theory, harmonic analysis, potential theory, partial differential equations, differential geometry. Since 1970, on the basis of the Dirac operator, Brackx et al. [9-12] etc. put forward the regular function, which is an extension of the holomorphic function in higher dimensional space. Thus they lay a theoretical foundation for Clifford analysis. The $k$-regular function is a natural generalization of the regular functions. In 1976, Brackx [13] was first to introduce $k$-regular functions of the real quaternion and gave the Cauchy integral formula and Taylor expansion. In 1977, Delanghe and Brackx [14] studied $k$-regular functions which were defined on $R^{n}$ with values in Clifford algebra space $\mathcal{A}_{n}(R)$ and obtained the corresponding Cauchy integral formula. Recently, many scholars such as Begehr et al. [15], Li et al. [16, 17], Zeng and Yang [18] etc. have studied some properties and corresponding boundary value problems of $k$-regular functions.

Teodorescu operator is the generalized solution of non-homogeneous Dirac equation. Therefore it plays a key role in studying the integral expression of non-homogeneous Dirac equation and many boundary value problems. In addition, it also have important applications in many subjects, such as physics, chemistry, engineering technology, etc. Due to its

(c) 2016 Wang. This article is distributed under the terms of the Creative Commons Attribution 4.0 International License (http://creativecommons.org/licenses/by/4.0/), which permits unrestricted use, distribution, and reproduction in any medium, provided you give appropriate credit to the original author(s) and the source, provide a link to the Creative Commons license, and indicate if changes were made. 
good properties and important applications, it has been a much studied and significant topic. Vekua [19] first discussed some properties of the Teodorescu operator detailedly, and Hile [20] studied some properties of the Teodorescu operator in $R^{n}$. Then Gilbert et al. [21] and Meng [22] studied its many properties in high dimensional complex space. Gürlebeck and Sprössig [23], and Yang [24] discussed its properties and the corresponding boundary value problems in the real quaternion analysis. Wang and Gong [25] discussed the stabilities of the singular integral operators when the boundary curve of integral domain is perturbed. Recently, Brackx et al. [26] studied some properties of the Teodorescu operator which is related to the Hermitian regular functions. Wang et al. [27, 28] showed some properties of the Teodorescu operator and corresponding boundary value problems.

In this paper, we define a kind of generalized Teodorescu operator which is related to the $k$-regular functions in real Clifford analysis. First, we discuss the boundedness and Hölder continuity for the generalized Teodorescu operator in a nonempty open bounded connected domain in $R^{n}$. Second, we discuss the stability and give the error estimate of the generalized Teodorescu operator when the boundary surface of the integral domain is perturbed. These results make the theory of Clifford analysis more perfect and also lay a theoretical foundation for studying the properties of singularity integral operator in Clifford analysis.

\section{Preliminaries}

Let $e_{1}, \ldots, e_{n}$ be an orthogonal basis of the Euclidean space $R^{n}$, and let $\mathcal{A}_{n}(R)$ be the Clifford algebra with basis $\left\{e_{A}: e_{A}=e_{\alpha_{1}} \cdots e_{\alpha_{h}}\right\}$, where $A=\left\{\alpha_{1}, \ldots, \alpha_{h}\right\} \subseteq\{1, \ldots, n\}, 1 \leq \alpha_{1}<\alpha_{2}<$ $\cdots<\alpha_{h} \leq n$ and $e_{\emptyset}=e_{0}=1$. The associative and noncommutative multiplication of the basis in $\mathcal{A}_{n}(R)$ is governed by the rules:

$$
\left\{\begin{array}{l}
e_{i}^{2}=-1, \quad i=1,2, \ldots, n \\
e_{i} e_{j}=-e_{j} e_{i}, \quad 1 \leq i, j \leq n, i \neq j \\
e_{0} e_{i}=e_{i}, \quad i=1,2, \ldots, n
\end{array}\right.
$$

Hence the real Clifford algebra is composed of elements having the type $a=\sum_{A} x_{A} e_{A}$, $x_{A} \in R$. The norm for an element $a \in \mathcal{A}_{n}(R)$ is taken to be $|a|=\sqrt{\sum_{A}\left|x_{A}\right|^{2}}$ and satisfies $|\bar{a}|=|a|,|a+b| \leq|a|+|b|,|a b| \leq 2^{n}|a||b|$.

In addition, we suppose $\Omega \subset R^{n}(n \geq 2)$ is a bounded connected domain and the boundary $\partial \Omega$ is a differentiable, oriented, and compact Liapunov surface. The function $f$, which is defined in $\Omega$ with values in $\mathcal{A}_{n}(R)$, can be expressed as $f(x)=\sum_{A} f_{A}(x) e_{A}$, where the $f_{A}(x)$ are real-valued functions. In this paper, let $f(x) \in C^{(r)}\left(\Omega, \mathcal{A}_{n}(R)\right)=\{f \mid f: \Omega \rightarrow$ $\left.\mathcal{A}_{n}(R), f(x)=\sum_{A} f_{A}(x) e_{A}\right\}$, where $f_{A}(x)$ has continuous $r$ times differentials. The Dirac operator is defined as follows:

$$
D f=\sum_{i=1}^{n} e_{i} \frac{\partial f}{\partial x_{i}}=\sum_{i=1}^{n} \sum_{A} e_{i} e_{A} \frac{\partial f_{A}}{\partial x_{i}}, \quad f D=\sum_{i=1}^{n} \frac{\partial f}{\partial x_{i}} e_{i}=\sum_{i=1}^{n} \sum_{A} e_{A} e_{i} \frac{\partial f_{A}}{\partial x_{i}}
$$

If $D f(x)=0(f(x) D=0)(x \in \Omega)$, then $f$ is called left(right) regular function. Furthermore, if $D^{k} f(x)=0\left(f(x) D^{k}=0\right)(x \in \Omega)$, where $r \geq k, k<n$. Then $f$ is called left (right) $k$-regular function. Usually left regular ( $k$-regular) is called regular ( $k$-regular) for short. And obviously, when $k=1$, a $k$-regular function is a regular function. $f(x) \in$ 
$L^{p}(\Omega)$ means $D^{j} f(x) \in L^{p}(\Omega), j=0,1, \ldots, k-1$, and $L_{p}[f(x), \Omega]=\sum_{j=0}^{k-1} L_{p}\left[D^{j} f(x), \Omega\right]$. Denote $B\left(\rho_{0}\right)=\left\{\omega \mid \omega=\sum_{i=1}^{n} \omega_{i}(x) e_{i}, \omega_{i} \in C^{1}(\partial \Omega), \omega_{n}(x) \geq 0,\|\omega\|_{\partial \Omega}<\rho_{0}\right\}$, where $\|\omega\|_{\partial \Omega}=$ $\max _{x \in \partial \Omega} \sqrt{\sum_{i=1}^{n} \sqrt{\sum_{j=1}^{n}\left|\frac{\partial\left(\omega_{i}(x)\right)}{\partial x_{j}}\right|^{2}}}+\max _{x \in \partial \Omega}|\omega(x)|$.

In the following, we define a kind of generalized singular Teodorescu operator and give some necessary lemmas. Then we discuss some properties of the generalized singular Teodorescu operator.

Definition 2.1 Let $\Omega$ be as stated above and $f(x) \in C^{(r)}\left(\Omega, \mathcal{A}_{n}(R)\right), D^{j} f(x) \in L^{p}(\Omega)(j=$ $0,1, \ldots, k-1)$, we define a kind of generalized singular Teodorescu operator as follows:

$$
\left(T_{\Omega}[f]\right)(y)=\sum_{j=0}^{k-1}(-1)^{j} \int_{\Omega} \frac{A_{j+1}}{\omega_{n}} \frac{D^{j} f(x)(\bar{x}-\bar{y})^{j+1}}{|x-y|^{n+\alpha}} d x
$$

where $0<\alpha<1$ is a positive constant and $\omega_{n}$ is the area of the unit sphere in $R^{n}, A_{j}$ is a constant as stated in [15], it is irrelevant to the $x, y$.

Remark 2.1 When $\alpha=0, n=2, k=1$, the singular integral operator is a normal Teodorescu operator.

Remark 2.2 When $y \in \Omega^{-}=R^{n}-\bar{\Omega}, T[f]$ is a normal generalized integral. When $y \in \bar{\Omega}$, $T[f]$ is a kind of generalized singular integral.

Lemma 2.1 ([2]) Let $\Omega \subset R^{n}(n \geq 2)$ be as stated above. When $\mu<n$, for any $y \in R^{n}$, we have $\int_{\Omega}|x-y|^{-\mu} d x \leq M_{3}$, where $M_{3}$ is a constant number which only depends on $\mu, \Omega$.

Lemma 2.2 ([16]) For any $x, y_{1}, y_{2} \in R^{n}$, when $j \geq 0$, we have

$$
\left|\frac{\left(x-y_{1}\right)^{j+1}}{\left|x-y_{1}\right|^{n}}-\frac{\left(x-y_{2}\right)^{j+1}}{\left|x-y_{2}\right|^{n}}\right| \leq\left[\sum_{i=1}^{n-1} \frac{\left(|x|+\left|y_{2}\right|\right)^{j}}{\left|x-y_{1}\right|^{i}\left|x-y_{2}\right|^{n-i}}+\sum_{i=1}^{j} \frac{\left(|x|+\left|y_{2}\right|\right)^{j-i}}{\left|x-y_{1}\right|^{n-i}}\right]\left|y_{1}-y_{2}\right| .
$$

$\operatorname{Remark}$ 2.3 When $j=0$, the second part $\sum_{i=1}^{j} \frac{\left(|x|+\left|y_{2}\right|\right)^{j-i}}{\left|x-y_{1}\right|^{n-i}}$ is vanishing.

Lemma 2.3 ([29]) If $\sigma_{1}, \sigma_{2}>0,0 \leq \alpha \leq 1$, then we have $\left|\sigma_{1}^{\alpha}-\sigma_{2}^{\alpha}\right| \leq\left|\sigma_{1}-\sigma_{2}\right|^{\alpha}$.

Lemma 2.4 ([21]) Suppose $\Omega$ is a bounded domain in $R^{n}(n \geq 2)$, and let $\alpha^{\prime}, \beta^{\prime}$ satisfy $0<\alpha^{\prime}, \beta^{\prime}<n, \alpha^{\prime}+\beta^{\prime}>n$. Then, for all $x_{1}, x_{2} \in R^{n}$ and $x_{1} \neq x_{2}$, we have

$$
\int_{\Omega}\left|t-x_{1}\right|^{-\alpha^{\prime}}\left|t-x_{2}\right|^{-\beta^{\prime}} d t \leq M_{1}\left(\alpha^{\prime}, \beta^{\prime}\right)\left|x_{1}-x_{2}\right|^{n-\alpha^{\prime}-\beta^{\prime}}
$$

Lemma 2.5 ([25]) Let $\Omega$ be as stated above. Suppose the area of $\partial \Omega$ is $A_{\partial \Omega}$ and $\partial \Omega_{\omega}=$ $\left\{t+\omega(t) \mid t \in \partial \Omega, \omega(t) \in B\left(\rho_{0}\right)\right\}$. Suppose $\Omega_{\omega}$ is the inside domain surrounded by $\partial \Omega_{\omega}$ and $\Omega_{\omega}^{-}=R^{n}-\Omega_{\omega}, E_{1}=\Omega_{\omega} \cap \Omega^{-}, E_{2}=\Omega_{\omega}^{-} \cap \Omega$. Then we have $A_{E_{1} \cup E_{2}} \leq M_{2}\|\omega\|_{\partial \Omega}$.

\section{Some properties of generalized Teodorescu operator}

Theorem 3.1 Let $\Omega \subset R^{n}(n \geq 2)$ be as stated above, $f(x) \in C^{(r)}\left(\Omega, \mathcal{A}_{n}(R)\right), D^{j} f(x) \in L^{p}(\Omega)$ $(j=0,1, \ldots, k-1), p>n /(1-\alpha)$. Then $T_{\Omega}[f](y)$ is uniformly bounded on $R^{n}$, and we have

$$
\left|T_{\Omega}[f](y)\right| \leq M_{4}(n, p, \alpha, \Omega) L_{p}[f, \Omega]
$$


Proof By the Hölder inequality, we have

$$
\begin{aligned}
\left|T_{\Omega}[f](y)\right| & =\left|\sum_{j=0}^{k-1}(-1)^{j} \int_{\Omega} \frac{A_{j+1}}{\omega_{n}} \frac{D^{j} f(x)(\bar{x}-\bar{y})^{j+1}}{|x-y|^{n+\alpha}} d x\right| \\
& \leq J_{1} \sum_{j=0}^{k-1}\left[\int_{\Omega}\left|D^{j} f(x)\right|^{p}|d x|\right]^{\frac{1}{p}}\left[\int_{\Omega} \frac{|d x|}{|x-y|^{(n+\alpha-j-1) q}}\right]^{\frac{1}{q}} \\
& \leq J_{2} L_{p}[f, \Omega] \sum_{j=0}^{k-1}\left[\int_{\Omega} \frac{|d x|}{|x-y|^{(n+\alpha-j-1) q}}\right]^{\frac{1}{q}} .
\end{aligned}
$$

Again from $p>n /(1-\alpha), 1 / p+1 / q=1$, we have $1<q<n /(n+\alpha-1)$. Thus

$$
(n+\alpha-j-1) q \leq(n+\alpha-1) q<n, \quad j=0,1, \ldots, k-1 .
$$

Hence for all $j=0,1, \ldots, k-1$, the integral $\int_{\Omega}|x-y|^{-(n+\alpha-j-1) q}|d x|$ is convergent. Thus it is bounded. And from Lemma 2.1, we can know its boundary is independent of $y$. Thus for all $y \in R^{n}$, we have

$$
\left|T_{\Omega}[f](y)\right| \leq M_{4}(n, p, \alpha, \Omega) L_{p}[f, \Omega]
$$

Theorem 3.2 Let $\Omega$ be as stated above, $f(x) \in C^{(r)}\left(\Omega, \mathcal{A}_{n}(R)\right), D^{j} f(x) \in L^{p}(\Omega)(j=0,1, \ldots$, $k-1), p>n /(1-\alpha)$. Then we have the following results:

(1) If $1 / 2 \leq \alpha<1$, then, for any $y_{1}, y_{2} \in \Omega$, we can obtain

$$
\left|T_{\Omega}[f]\left(y_{1}\right)-T_{\Omega}[f]\left(y_{2}\right)\right| \leq M_{5}(n, p, \alpha, \Omega) L_{p}[f, \Omega]\left|y_{1}-y_{2}\right|^{\beta}
$$

where $\beta=1-\alpha-n / p, 0<\beta<1$.

(2) If $0<\alpha<1 / 2$, let $p_{1}$ be a constant and satisfies $p_{1}<p, n /(1-\alpha)<p_{1}<n /(1-2 \alpha)$, then, for any $y_{1}, y_{2} \in \Omega$, we have

$$
\left|T_{\Omega}[f]\left(y_{1}\right)-T_{\Omega}[f]\left(y_{2}\right)\right| \leq M_{6}(n, p, \alpha, \Omega) L_{p_{1}}[f, \Omega]\left|y_{1}-y_{2}\right|^{\gamma},
$$

where $\gamma=1-\alpha-n / p_{1}, 0<\gamma<1$.

Proof (1) From Definition 2.1, we get

$$
\begin{aligned}
& \left|T_{\Omega}[f]\left(y_{1}\right)-T_{\Omega}[f]\left(y_{2}\right)\right| \\
& =\mid \sum_{j=0}^{k-1}(-1)^{j} \int_{\Omega} \frac{A_{j+1}}{\omega_{n}} \frac{D^{j} f(x)\left(\bar{x}-\bar{y}_{1}\right)^{j+1}}{\left|x-y_{1}\right|^{n+\alpha}} d x \\
& \quad-\sum_{j=0}^{k-1}(-1)^{j} \int_{\Omega} \frac{A_{j+1}}{\omega_{n}} \frac{D^{j} f(x)\left(\bar{x}-\bar{y}_{2}\right)^{j+1}}{\left|x-y_{2}\right|^{n+\alpha}} d x \mid \\
& \leq J_{3} \sum_{j=0}^{k-1} \int_{\Omega}\left|D^{j} f(x)\right|\left|\frac{\left(\bar{x}-\bar{y}_{1}\right)^{j+1}}{\left|x-y_{1}\right|^{n+\alpha}}-\frac{\left(\bar{x}-\bar{y}_{2}\right)^{j+1}}{\left|x-y_{2}\right|^{n+\alpha}}\right||d x| .
\end{aligned}
$$


Again, from Lemma 2.2, Lemma 2.3, and $\Omega$ being a bounded domain, we obtain

$$
\begin{aligned}
&\left|\frac{\left(\bar{x}-\bar{y}_{1}\right)^{j+1}}{\left|x-y_{1}\right|^{n+\alpha}}-\frac{\left(\bar{x}-\bar{y}_{2}\right)^{j+1}}{\left|x-y_{2}\right|^{n+\alpha}}\right| \\
& \leq \frac{1}{\left|x-y_{1}\right|^{\alpha}}\left[\sum_{i=1}^{n-1} \frac{\left(|x|+\left|y_{2}\right|\right)^{j}}{\left|x-y_{1}\right|^{i}\left|x-y_{2}\right|^{n-i}}+\sum_{i=1}^{j} \frac{\left(|x|+\left|y_{2}\right|\right)^{j-i}}{\left|x-y_{1}\right|^{n-i}}\right]\left|y_{1}-y_{2}\right| \\
&+\frac{1}{\left|x-y_{2}\right|^{n-j-1}}\left|\frac{1}{\left|x-y_{1}\right|}-\frac{1}{\left|x-y_{2}\right|}\right|^{\alpha} \\
& \leq \frac{1}{\left|x-y_{1}\right|^{\alpha}} \sum_{i=1}^{n-1} \frac{\left(|x|+\left|y_{2}\right|\right)^{j}}{\left|x-y_{1}\right|^{i}\left|x-y_{2}\right|^{n-i}}\left|y_{1}-y_{2}\right| \\
&+\frac{1}{\left|x-y_{1}\right|^{\alpha}} \sum_{i=1}^{j} \frac{\left(|x|+\left|y_{2}\right|\right)^{j-i}}{\left|x-y_{1}\right|^{n-i}}\left|y_{1}-y_{2}\right|+\frac{1}{\left|x-y_{2}\right|^{n-j-1}} \frac{\left|y_{1}-y_{2}\right|^{\alpha}}{\left|x-y_{1}\right|^{\alpha}\left|x-y_{2}\right|^{\alpha}} \\
& \leq J_{4} \frac{1}{\left|x-y_{1}\right|^{\alpha}} \sum_{i=1}^{n-1} \frac{1}{\left|x-y_{1}\right|^{i}\left|x-y_{2}\right|^{n-i}}\left|y_{1}-y_{2}\right| \\
& \quad+J_{5} \frac{1}{\left|x-y_{1}\right|^{\alpha}} \sum_{i=1}^{j} \frac{1}{\left|x-y_{1}\right|^{n-i}}\left|y_{1}-y_{2}\right|+\frac{\left|y_{1}-y_{2}\right|^{\alpha}}{\left|x-y_{1}\right|^{\alpha}\left|x-y_{2}\right|^{n+\alpha-j-1}} .
\end{aligned}
$$

Thus, from the above, we get

$$
\begin{aligned}
& \left|T_{\Omega}[f]\left(y_{1}\right)-T_{\Omega}[f]\left(y_{2}\right)\right| \\
& \leq J_{6} \sum_{j=0}^{k-1} \sum_{i=1}^{n-1} \int_{\Omega} \frac{1}{\left|x-y_{1}\right|^{i+\alpha}\left|x-y_{2}\right|^{n-i}}\left|D^{j} f(x)\right||d x|\left|y_{1}-y_{2}\right| \\
& \quad+J_{7} \sum_{j=1}^{k-1} \sum_{i=1}^{j} \int_{\Omega} \frac{1}{\left|x-y_{1}\right|^{n+\alpha-i}}\left|D^{j} f(x)\right||d x|\left|y_{1}-y_{2}\right| \\
& \quad+J_{8} \sum_{j=0}^{k-1} \int_{\Omega} \frac{1}{\left|x-y_{1}\right|^{\alpha}\left|x-y_{2}\right|^{n+\alpha-j-1}}\left|D^{j} f(x)\right||d x|\left|y_{1}-y_{2}\right|^{\alpha} \\
& =I_{1}+I_{2}+I_{3} .
\end{aligned}
$$

In the following, first we evaluate $I_{1}$. From the Hölder inequality, we have

$$
\begin{aligned}
I_{1} & \leq J_{9} L_{p}[f, \Omega] \sum_{i=1}^{n-1}\left[\int_{\Omega}\left|x-y_{1}\right|^{-(i+\alpha) q}\left|x-y_{2}\right|^{-(n-i) q}|d x|\right]^{\frac{1}{q}} \cdot\left|y_{1}-y_{2}\right| \\
& =J_{9} L_{p}[f, \Omega]\left|y_{1}-y_{2}\right| \sum_{i=1}^{n-1} I_{1 i}^{\frac{1}{q}},
\end{aligned}
$$

where $I_{1 i}=\int_{\Omega}\left|x-y_{1}\right|^{-(i+\alpha) q}\left|x-y_{2}\right|^{-(n-i) q}|d x|, i=1,2, \ldots, n-1$.

In addition, when $p>n /(1-\alpha)$, we have $1<q<n /(n+\alpha-1)$. Thus let $\alpha^{\prime}=(i+\alpha) q$, $\beta^{\prime}=(n-i) q$, then

$$
0<\alpha^{\prime}=(i+\alpha) q<(i+\alpha) \frac{n}{n+\alpha-1} \leq \frac{(n-1+\alpha) n}{n+\alpha-1}=n,
$$




$$
0<\beta^{\prime}=(n-i) q<(n-i) \frac{n}{n+\alpha-1} \leq \frac{n(n-1)}{n+\alpha-1}<n .
$$

And $\alpha^{\prime}+\beta^{\prime}=(n+\alpha) q>(n+\alpha)>n$.

Thus from Lemma 2.4, we have

$$
I_{1 i} \leq J_{10}\left|y_{1}-y_{2}\right|^{n-\alpha^{\prime}-\beta^{\prime}}=J_{10}\left|y_{1}-y_{2}\right|^{n-(n+\alpha) q}, \quad i=1,2, \ldots, n-1
$$

So by (3.4), we know

$$
I_{1} \leq J_{9} L_{p}[f, \Omega]\left|y_{1}-y_{2}\right| \sum_{i=1}^{n-1} J_{10}^{\frac{1}{q}}\left|y_{1}-y_{2}\right|^{\frac{n-(n+\alpha) q}{q}} \leq J_{11} L_{p}[f, \Omega]\left|y_{1}-y_{2}\right|^{\beta}
$$

where $\beta=1-\alpha-n / p$, and from $p>n /(1-\alpha)$, we have $0<\beta=1-\alpha-n / p=1-\alpha-n / p<1$.

In addition

$$
\begin{aligned}
I_{2} \leq & J_{12} \sum_{j=1}^{k-1} \sum_{i=1}^{j}\left[\int_{\Omega}\left|D^{j} f(x)\right|^{p}|d x|\right]^{\frac{1}{p}}\left[\int_{\Omega} \frac{|d x|}{\left|x-y_{1}\right|^{(n+\alpha-i) q}}\right]^{\frac{1}{q}}\left|y_{1}-y_{2}\right| \\
= & J_{12}\left|y_{1}-y_{2}\right|\left\{\left[\int_{\Omega}|D f(x)|^{p} \mid d x\right]^{\frac{1}{p}}\left[\int_{\Omega} \frac{|d x|}{\left|x-y_{1}\right|^{(n+\alpha-1) q}}\right]^{\frac{1}{q}}\right. \\
& +\sum_{i=1}^{2}\left[\int_{\Omega}\left|D^{2} f(x)\right|^{p}|d x|\right]^{\frac{1}{p}}\left[\int_{\Omega} \frac{|d x|}{\left|x-y_{1}\right|^{(n+\alpha-i) q}}\right]^{\frac{1}{q}} \\
& +\cdots \\
& \left.+\sum_{i=1}^{k-1}\left[\int_{\Omega}\left|D^{k-1} f(x)\right|^{p}|d x|\right]^{\frac{1}{p}}\left[\int_{\Omega} \frac{|d x|}{\left|x-y_{1}\right|^{(n+\alpha-i) q}}\right]^{\frac{1}{q}}\right\} .
\end{aligned}
$$

Again from $1<q<n /(n+\alpha-1)$. Thus, for all $1 \leq i \leq k-1(k<n)$, we have

$$
(n+\alpha-i) q<\frac{(n+\alpha-i) n}{(n+\alpha-1)} \leq n
$$

Thus, for all $i=1,2, \ldots, k-1(k<n)$, the integral $\int_{\Omega}\left|x-y_{1}\right|^{-(n+\alpha-i) q}|d x|$ is convergent. So we obtain

$$
I_{2} \leq J_{13} L_{p}[f, \Omega]\left|y_{1}-y_{2}\right| \leq J_{14} L_{p}[f, \Omega]\left|y_{1}-y_{2}\right|^{\beta}
$$

And again from the Hölder inequality, we have

$$
\begin{aligned}
I_{3} & \leq J_{15} L_{p}[f, \Omega]\left|y_{1}-y_{2}\right|^{\alpha} \sum_{j=0}^{k-1}\left[\int_{\Omega} \frac{|d x|}{\left|x-y_{1}\right|^{\alpha q}\left|x-y_{2}\right|^{(n+\alpha-j-1) q}}\right]^{\frac{1}{q}} \\
& =J_{15} L_{p}[f, \Omega]\left|y_{1}-y_{2}\right|^{\alpha} \sum_{j=0}^{k-1} I_{3 j}^{\frac{1}{q}},
\end{aligned}
$$

where $I_{3 j}=\int_{\Omega}\left|x-y_{1}\right|^{-\alpha q}\left|x-y_{2}\right|^{-(n+\alpha-j-1) q}|d x|$. 
When $j=0, I_{30}=\int_{\Omega}\left|x-y_{1}\right|^{-\alpha q}\left|x-y_{2}\right|^{-(n+\alpha-1) q}|d x|$. Let $\alpha^{\prime}=\alpha q, \beta^{\prime}=(n+\alpha-1) q$. From $1<q<n /(n+\alpha-1)$, we get

$$
\begin{aligned}
& 0<\alpha^{\prime}=\alpha q<\frac{\alpha n}{n+\alpha-1}<n, \\
& 0<\beta^{\prime}=(n+\alpha-1) q<n .
\end{aligned}
$$

Again from $1 / 2 \leq \alpha<1$, we have

$$
\alpha^{\prime}+\beta^{\prime}=(n+2 \alpha-1) q>n q>n
$$

Thus by Lemma 2.4, we obtain

$$
I_{30} \leq J_{16}\left|y_{1}-y_{2}\right|^{n-\alpha^{\prime}-\beta^{\prime}}=J_{16}\left|y_{1}-y_{2}\right|^{n-(n+2 \alpha-1) q} .
$$

When $j \neq 0$, that is, $j=1,2, \ldots, k-1, k<n$,

$$
\alpha q+(n+\alpha-j-1) q<\frac{(n+\alpha-1+\alpha-j) n}{n+\alpha-1}=\left(1+\frac{\alpha-j}{n+\alpha-1}\right) n<n .
$$

So $I_{3 j}(j=1,2, \ldots, k-1, k<n)$ is convergent, thus there is no harm to suppose $I_{3 j} \leq J_{17}$. Therefore from the above, we obtain

$$
\begin{aligned}
I_{3} & \leq J_{15} L_{p}[f, \Omega]\left|y_{1}-y_{2}\right|^{\alpha}\left[I_{30}^{\frac{1}{q}}+\sum_{j=1}^{k-1} I_{3 j}^{\frac{1}{q}}\right] \\
& =J_{15} L_{p}[f, \Omega]\left|y_{1}-y_{2}\right|^{\alpha}\left[J_{16}^{\frac{1}{q}}\left|y_{1}-y_{2}\right|^{\frac{n-(n+2 \alpha-1) q}{q}}+(k-1) J_{17}^{\frac{1}{q}}\right] \\
& \leq J_{18} L_{p}[f, \Omega]\left|y_{1}-y_{2}\right|^{\alpha+\frac{n-(n+2 \alpha-1) q}{q}}+J_{19} L_{p}[f, \Omega]\left|y_{1}-y_{2}\right|^{\alpha} \\
& =J_{18} L_{p}[f, \Omega]\left|y_{1}-y_{2}\right|^{1-\alpha-\frac{n}{p}}+J_{19} L_{p}[f, \Omega]\left|y_{1}-y_{2}\right|^{2 \alpha-1-\frac{n}{p}}\left|y_{1}-y_{2}\right|^{1-\alpha-\frac{n}{p}}
\end{aligned}
$$

Again when $1 / 2 \leq \alpha<1$, we know $\alpha-(1-\alpha)-n / p=2 \alpha-1+n / p>0$ and $\Omega$ is bounded. Thus we know that $\left|y_{1}-y_{2}\right|^{2 \alpha-1-n / p}$ is bounded. So we have

$$
I_{3} \leq J_{20} L_{p}[f, \Omega]\left|y_{1}-y_{2}\right|^{1-\alpha-n / p}=J_{20} L_{p}[f, \Omega]\left|y_{1}-y_{2}\right|^{\beta},
$$

where $\beta=1-\alpha-n / p$.

Thus from (3.3), (3.5), (3.6), and (3.7), we can get

$$
\left|T_{\Omega}[f]\left(y_{1}\right)-T_{\Omega}[f]\left(y_{2}\right)\right| \leq\left(J_{11}+J_{14}+J_{20}\right) L_{p}[f, \Omega]\left|y_{1}-y_{2}\right|^{\beta}=J_{21} L_{p}[f, \Omega]\left|y_{1}-y_{2}\right|^{\beta} .
$$

In addition, from the above proof process, we know that $J_{21}$ is only dependent on $n, p$, $\alpha, \Omega$. Taking $M_{5}=J_{21}$, then, when $1 / 2 \leq \alpha<1$, for all $y_{1}, y_{2} \in \Omega$, we can obtain

$$
\left|T_{\Omega}[f]\left(y_{1}\right)-T_{\Omega}[f]\left(y_{2}\right)\right| \leq M_{5}(n, p, \alpha, \Omega) L_{p}[f, \Omega]\left|y_{1}-y_{2}\right|^{\beta}
$$

(2) When $0<\alpha<1 / 2$, because of $p_{1}<p, f \in L^{p}(\Omega)$, we know $f \in L^{p_{1}}(\Omega)$. Let $q_{1}$ satisfies $1 / p_{1}+1 / q_{1}=1$. From $n /(1-\alpha)<p_{1}<n /(1-2 \alpha)$, thus we get $n /(n+2 \alpha-1)<q_{1}<n /(n+\alpha-1)$. 
So similar to the text in front of the discussion of $I_{1}$, obviously, we can get $0<\alpha^{\prime}, \beta^{\prime}<n$ and $\alpha^{\prime}+\beta^{\prime}=(n+\alpha) q_{1}>n$.

Thus from Lemma 2.4, we have

$$
I_{1} \leq J_{11}^{\prime} L_{p_{1}}[f, \Omega]\left|y_{1}-y_{2}\right|^{\gamma}
$$

where $\gamma=1-\alpha-n / p_{1}, 0<\gamma<1$.

Again completely analogous to the discussion in (1), we have

$$
I_{2} \leq J_{14}^{\prime} L_{p_{1}}[f, \Omega]\left|y_{1}-y_{2}\right|^{\gamma}
$$

In addition, similar to the text in front of the discussion of $I_{3}$, we have

$$
I_{3} \leq J_{15}^{\prime} L_{p_{1}}[f, \Omega]\left|y_{1}-y_{2}\right|^{\alpha} \sum_{j=0}^{k-1} I_{3 j}^{\frac{1}{q_{1}}},
$$

where $I_{3 j}=\int_{\Omega}\left|x-y_{1}\right|^{-\alpha q_{1}}\left|x-y_{2}\right|^{-(n+\alpha-j-1) q_{1}}|d x|$.

When $j=0,0<\alpha^{\prime}=\alpha q_{1}<n, 0<\beta^{\prime}=(n+\alpha-1) q_{1}<n$. Thus

$$
\alpha^{\prime}+\beta^{\prime}=(n+2 \alpha-1) q_{1}>(n+2 \alpha-1) \frac{n}{n+2 \alpha-1}=n .
$$

Thus from Lemma 2.4, we can get

$$
I_{30} \leq J_{16}^{\prime}\left|y_{1}-y_{2}\right|^{n-(n+2 \alpha-1) q_{1}} .
$$

When $j \neq 0$, again completely analogous to the discussion in (1), we know $I_{3 j}$ is convergent. So there is no harm to suppose $I_{3 j} \leq J_{17}^{\prime}$.

Thus, similarly, we have

$$
I_{3} \leq J_{20}^{\prime} L_{p_{1}}[f, \Omega]\left|y_{1}-y_{2}\right|^{\gamma} .
$$

Therefore from (3.3), (3.8), (3.9), and (3.10), we obtain

$$
\left|T_{\Omega}[f]\left(y_{1}\right)-T_{\Omega}[f]\left(y_{2}\right)\right| \leq J_{21}^{\prime} L_{p_{1}}[f, \Omega]\left|y_{1}-y_{2}\right|^{\gamma} .
$$

Taking $M_{6}=J_{21}^{\prime}$, we can get

$$
\left|T_{\Omega}[f]\left(y_{1}\right)-T_{\Omega}[f]\left(y_{2}\right)\right| \leq M_{6}(n, p, \alpha, \Omega) L_{p_{1}}[f, \Omega]\left|y_{1}-y_{2}\right|^{\gamma} .
$$

\section{The stability and error estimate of generalized Teodorescu operator}

Theorem 4.1 Let $\Omega$ be as stated above, $f(x) \in C^{(r)}\left(\Omega, \mathcal{A}_{n}(R)\right), D^{j} f(x) \in L^{p}(\Omega)(j=0,1, \ldots$, $k-1), p>n /(1-\alpha), y \in \Omega, \rho_{0}$ is a given constant, and $\omega \in B\left(\rho_{0}\right)$. Then we have

$$
\left|\left(T_{\Omega_{\omega}}[f]\right)(y)-\left(T_{\Omega}[f]\right)(y)\right| \leq M_{7}(n, p, \alpha, \Omega) L_{p}[f, \Omega]\|\omega\|_{\partial \Omega}^{\gamma},
$$

where $\gamma=(1-\alpha) / n-1 / p>0$. 
Proof First

$$
\begin{aligned}
& \left|\left(T_{\Omega_{\omega}}[f]\right)(y)-\left(T_{\Omega}[f]\right)(y)\right| \\
& \quad=\left|\sum_{j=0}^{k-1} \int_{E_{1}} \frac{A_{j+1}}{\omega_{n}} \frac{D^{j} f(x)(\bar{x}-\bar{y})^{j+1}}{|x-y|^{n+\alpha}} d x-\sum_{j=0}^{k-1} \int_{E_{2}} \frac{A_{j+1}}{\omega_{n}} \frac{D^{j} f(x)(\bar{x}-\bar{y})^{j+1}}{|x-y|^{n+\alpha}} d x\right| \\
& \quad \leq\left|\sum_{j=0}^{k-1} \int_{E_{1}} \frac{A_{j+1}}{\omega_{n}} \frac{D^{j} f(x)(\bar{x}-\bar{y})^{j+1}}{|x-y|^{n+\alpha}} d x\right|+\left|\sum_{j=0}^{k-1} \int_{E_{2}} \frac{A_{j+1}}{\omega_{n}} \frac{D^{j} f(x)(\bar{x}-\bar{y})^{j+1}}{|x-y|^{n+\alpha}} d x\right| \\
& \quad=I_{1}+I_{2},
\end{aligned}
$$

where $E_{1}, E_{2}$ are the domains as in Lemma 2.5.

Then from $\Omega, E_{1}$ being a bounded domain, $|x-y|^{j q}$ is bounded. Again by the Hölder inequality, we have

$$
\begin{aligned}
I_{1} & \leq J_{22} \sum_{j=0}^{k-1}\left[\int_{E_{1}}\left|D^{j} f(x)\right|^{p}|d x|\right]^{\frac{1}{p}}\left[\int_{E_{1}} \frac{|x-y|^{(j+1) q}}{|x-y|^{(n+\alpha) q}}|d x|\right]^{\frac{1}{q}} \\
& \leq J_{23} L_{p}[f, \Omega] \sum_{j=0}^{k-1}\left[\int_{E_{1}} \frac{|x-y|^{\mid q q}}{|x-y|^{(n+\alpha-1) q}}|d x|\right]^{\frac{1}{q}} \\
& \leq J_{24} L_{p}[f, \Omega] \sum_{j=0}^{k-1}\left[\int_{E_{1}} \frac{1}{|x-y|^{(n+\alpha-1) q}}|d x|\right]^{\frac{1}{q}} \\
& \leq J_{25} L_{p}[f, \Omega]\left[\int_{E_{1}} \frac{1}{|x-y|^{(n+\alpha-1) q}}|d x|^{\frac{1}{q}} .\right.
\end{aligned}
$$

Taking $E_{11}=E_{1} \cap\left\{x|| x-y \mid \geq\|\omega\|_{\partial \Omega}^{\frac{1}{n}}\right\}, E_{12}=E_{1} \cap\left\{x|| x-y \mid<\|\omega\|_{\partial \Omega}^{\frac{1}{n}}\right\}$, then obviously $E_{1}=E_{11} \cup E_{12}, E_{11} \cap E_{12}=\emptyset$. Thus

$$
\begin{aligned}
& {\left[\int_{E_{1}} \frac{1}{|x-y|^{(n+\alpha-1) q}}|d x|\right]^{\frac{1}{q}}} \\
& \quad=\left[\int_{E_{11}} \frac{1}{|x-y|^{(n+\alpha-1) q}}|d x|+\int_{E_{12}} \frac{1}{|x-y|^{(n+\alpha-1) q}}|d x|\right]^{\frac{1}{q}} \\
& \quad \leq\left[\int_{E_{11}} \frac{1}{\|\omega\|_{\partial \Omega}^{\frac{1}{n}(n+\alpha-1) q}}|d x|+\int_{E_{12}} \frac{1}{|x-y|^{(n+\alpha-1) q}} \mid d x\right]^{\frac{1}{q}} .
\end{aligned}
$$

In addition, from Lemma 2.5, we have

$$
\begin{aligned}
& \int_{E_{11}} \frac{1}{\|\omega\|_{\partial \Omega}^{\frac{1}{n}(n+\alpha-1) q}}|d x| \\
& \quad=\|\omega\|_{\partial \Omega}^{\frac{1}{n}(n+\alpha-1) q} A_{E_{11}} \leq\|\omega\|_{\partial \Omega}^{-\frac{1}{n}(n+\alpha-1) q} A_{E_{11} \cup E_{12}} \\
& \quad \leq C\|\omega\|_{\partial \Omega}^{\left[1-\frac{1}{n}(n+\alpha-1) q\right]}=C\|\omega\|_{\partial \Omega}^{\frac{1}{n}[n-n q+(1-\alpha) q]} .
\end{aligned}
$$


Again when $p>n /(1-\alpha), 1<q<n /(n+\alpha-1)$. Thus $(n+\alpha-1) q-(n-1)<1$. So from the local generalized spherical coordinate, we obtain

$$
\begin{aligned}
& \int_{E_{12}} \frac{1}{|x-y|^{(n+\alpha-1) q}}|d x| \\
& \quad \leq J_{26} \int_{0}^{\|\omega\|_{\partial \Omega}^{\frac{1}{n}}} \frac{1}{\rho_{0}^{[(n+\alpha-1) q-(n-1)]}} d \rho_{0}=J_{26}\|\omega\|_{\partial \Omega}^{\frac{1}{n}[n-n q+(1-\alpha) q]} .
\end{aligned}
$$

Thus from (4.2)-(4.5) and $1 / p+1 / q=1$, we get

$$
\begin{aligned}
I_{1} & \leq J_{25} L_{p}[f, \Omega]\left[C\|\omega\|_{\partial \Omega}^{\frac{1}{\eta}[n-n q+(1-\alpha) q]}+J_{26}\|\omega\|_{\partial \Omega}^{\frac{1}{n}[n-n q+(1-\alpha) q]}\right]^{\frac{1}{q}} \\
& \leq J_{27} L_{p}[f, \Omega]\|\omega\|_{\partial \Omega}^{\frac{n-n q+(1-\alpha) q}{n q}} \\
& =J_{27} L_{p}[f, \Omega]\|\omega\|_{\partial \Omega}^{\frac{(1-\alpha) p-n}{n p}} \\
& =J_{27} L_{p}[f, \Omega]\|\omega\|_{\partial \Omega}^{\gamma}
\end{aligned}
$$

where $\gamma=(1-\alpha) / n-1 / p>0$.

Similarly, we have

$$
I_{2} \leq J_{28} L_{p}[f, \Omega]\|\omega\|_{\partial \Omega}^{\gamma}
$$

Therefore from (4.1), (4.6), and (4.7), we obtain

$$
\left|\left(T_{\Omega_{\omega}}[f]\right)(y)-\left(T_{\Omega}[f]\right)(y)\right| \leq J_{29} L_{p}[f, \Omega]\|\omega\|_{\partial \Omega}^{\gamma} .
$$

We take $M_{7}=J_{29}$, that is,

$$
\left|\left(T_{\Omega_{\omega}}[f]\right)(y)-\left(T_{\Omega}[f]\right)(y)\right| \leq M_{7} L_{p}[f, \Omega]\|\omega\|_{\partial \Omega}^{\gamma} .
$$

From the above proof process, we know that $M_{7}=J_{29}$ is only dependent on $n, p, \alpha, \Omega$.

\section{Competing interests}

The author declares that they have no competing interests.

\section{Author's contributions}

LW has done all contributions to the article.

\section{Acknowledgements}

This work was supported by the National Science Foundation of China (No. 11401162, No. 11571089, No. 11401159, No. 11301136) and the Natural Science Foundation of Hebei Province (No. A2015205012, No. A2014205069, No. A2014208158) and Hebei Normal University Doctor Fund (No. L2015B03).

Received: 28 November 2015 Accepted: 19 March 2016 Published online: 31 March 2016

\section{References}

1. Clifford, WK: Applications of Grassmann's extensive algebra. Am. J. Math. 1, 350-358 (1878)

2. Gilbert, RP: First Order Elliptic Systems: A Function Theoretic Approach. Academic Press, New York (1983)

3. Zaharia, MD, Dorst, L: Modeling and visualization of 3D polygonal mesh surfaces using geometric algebra. Comput. Graph. 28, 519-526 (2004)

4. Batard, T, Saint-Jean, C, Berthier, M: A metric approach to nD images edge detection with Clifford algebras. J. Math. Imaging Vis. 33, 296-312 (2009) 
5. Xu, C, Liu, H, Cao, WM, Feng, JQ: Multispectral image edge detection via Clifford gradient. Sci. China, Inf. Sci. 55, 260-269 (2012)

6. Rivera-Rrovelo, J, Bayro-Corrochano, E: Medical image segmentation using a self-organizing neural network. In: International Joint Conference on Neural Networks, pp. 3538-3545 (2006)

7. Bayro-Corrochano, E, Vallejo, R, Arana-Daniel, N: Geometric preprocessing, geometric feedforward neural networks and Clifford support vector machines for visual learning. Neurocomputing 67, 54-105 (2005)

8. Ryan, J: Clifford Algebra and Their Applications in Mathematical Physics. Volume 2: Clifford Analysis. Birkhäuser, Basel (1996)

9. Brackx, F, Delanghe, R, Sommen, F: Clifford Analysis. Research Notes in Mathematics. Pitman, Boston (1982)

10. Delanghe, R: On regular analytic functions with values in Clifford algebra. Math. Ann. 185, 91-111 (1970)

11. Delanghe, R: On the singularities of functions with values in Clifford algebra. Math. Ann. 196, 293-319 (1972)

12. Sommen, F: Spherical monogenic functions and analytic functions on the unit sphere. Tokyo J. Math. 4, 427-456 (1981)

13. Brackx, F: On (k)-monogenic functions of a quaternion variable. In: Function Theoretic Methods in Differential Equations. Research Notes in Mathematics, vol. 8, pp. 22-44 (1976)

14. Delanghe, R, Brackx, F: Hypercomplex function theory and Hilbert modules with reproducing kernel. Proc. Lond. Math. Soc. 37, 545-576 (1978)

15. Begehr, H, Du, JY, Zhang, ZX: On higher order Cauchy-Pompeiu formula in Clifford analysis and its application. Gen. Math. 11, 5-26 (2003)

16. $\mathrm{Li}, \mathrm{XL}, \mathrm{Qiao}, \mathrm{YY}, \mathrm{Hu}, \mathrm{WW}$, Wang, $\mathrm{HY}$ : Some properties of the $k$-regular functions and boundary behavior of the higher order Cauchy-type integral in Clifford analysis. Adv. Math. 39, 31-48 (2010)

17. Qiao, YY, Yang, HJ, Li, XL: Some properties for k-biregular function in Clifford analysis. Adv. Math. 41, 187-198 (2012)

18. Zeng, W, Yang, PW: Riemann boundary value problems of non-normal type for $k$-regular functions. J. Sichuan Norm. Univ. Nat. Sci. 30, 291-294 (2007)

19. Vekua, IN: Generalized Analytic Functions. Pergamon, Oxford (1962)

20. Hile, GN: Elliptic systems in the plane with first-order terms and constant coefficients. Commun. Partial Differ. Equ. 3 , 949-977 (1978)

21. Gilbert, RP, Hou, ZY, Meng, XW: Vekua theory in higher-dimensional complex spaces: the Il-operator in $\mathbb{C}^{n}$. Complex Var. 21, 99-105 (1993)

22. Meng, XW: T-Operator in $\mathbb{C}^{n}$ and its applications. In: Integral Equations and Boundary Value Problems, pp. 147-152. World Scientific, Singapore (1991)

23. Gürlebeck, K, Sprössig, W: Quaternionic Analysis and Elliptic Boundary Value Problems. Birkhäuser, Basel (1990)

24. Yang, PW: $H_{\lambda}$-Regular vector functions and their boundary value problems. Bound. Value Probl. 2012, 75 (2012)

25. Wang, XL, Gong, YF: The stabilities of Cauchy kernel integral about the integral surface. Chin. Ann. Math., Ser. A 22, 447-452 (2001)

26. Brackx, F, De Schepper, H, Luna-Elizarrarás, ME, Shapiro, M: The Teodorescu operator in Clifford analysis. Chin. Ann. Math., Ser. B 33, 625-640 (2012)

27. Wang, LP, Qiao, YY, Yang, HJ: Some properties of the Teodorescu operator related to the $\alpha$-Dirac operator. Appl. Anal. 93, 2413-2425 (2014)

28. Wang, LP, Xu, ZL, Qiao, YY: The mixed boundary value problem for the inhomogeneous Cimmino system. Bound. Value Probl. 2015, 13 (2015)

29. Zhao, Z: Singular Integral Equation. Beijing Normal University Press, Beijing (1984) (in Chinese)

\section{Submit your manuscript to a SpringerOpen ${ }^{\circ}$ journal and benefit from:}

- Convenient online submission

Rigorous peer review

- Immediate publication on acceptance

- Open access: articles freely available online

- High visibility within the field

- Retaining the copyright to your article 